\title{
Electron Holographic and Lorentz Microscopic I mages of Variously Shaped Magnetic Specimens
}

\author{
K. Yamamoto and T. Hirayama*
}

Japan Society for the Promotion of Science, Domestic Research Fellow, c/o Japan Fine Ceramics Center, 2-4-1, Mutsuno, Atsuta, Nagoya, Japan, 456-8587

* Japan Fine Ceramics Center, 2-4-1, Mutsuno, Atsuta, Nagoya, Japan, 456-8587

L orentz microscopy and el ectron holography have been used to observe magnetic-domain structures. In order to interpret these images, such experimentally observed images should be compared with simulated images. In particular, to correctly interpret phase images obtained by electron holography, it is necessary to simulate the images with consideration for specimen inner potential as well as magnetic fields. A few methods for simulating magnetic flux images or Lorentz images of thin magnetic films have been proposed [1,2]. However, it is difficult to calculate these images of wedge-shaped specimens or more complicated shapes. In light of these problems, we have devel oped a new method for simulating holographic images and Lorentz images of variously shaped magnetic specimens.

To simulate holographic images or Lorentz images, firstly, a model of the magnetic domain structures is required, and its object wave, namely, the wave function bel ow the specimen must be cal culated. For a single magnetic-domain spherical particle, we have al ready devised al gorithm that considers not only the inner potential but also the amplitude modulation due to electron absorption for calculating the object wave [3]. It is assumed here that the magnetic specimen is composed of single magnetic-domain particles. By putting the particles in the specimen model along the directions of magnetic vectors as shown in Fig. 1, and by integrating each amplitude or phase, the object wave of the whole magnetic specimen can be cal culated.

Figure 2 shows the interference micrograph of the wedge-shaped specimen model illustrated in Fig. 1. The magnetic-flux distributions not only on the inside of the specimen but also on the outside were simulated by the new method. Simulated under-focused Lorentz image and over-focused image are shown in Fig. 3(a) and 3(b). White lines corresponding to the magnetic walls were observed. However, black lines were not observed clearly, because the black contrast was buried in the amplitude modulation caused by electron absorption. Figure 4 shows the phase image of a cylindrical rod specimen. The direction of the arrow indicates that of the magnetic vector. This simulation is useful for analyzing the experimental images of a magnetic nano-wire.

In conclusion, we have devel oped the new method for simulating the holographic images or Lorentz 
images of any shape of specimens or any type of magnetic-domain structures. At the conference, we will present some simulated holograms of the above specimen models, and compare the simulated images with experimentally observed images.

\section{References}

[1] M.Beleggia et al., Proceedings of $15^{\text {th }}$ International Congress on Electron Microscopy, 1, (2002) 307-308.

[2] T.Haug et al., Proceedings of $15^{\text {th }}$ International Congress on Electron Microscopy, 1, (2002) 321-322.

[3] K.Yamamoto et al., Ultramicroscopy 85, (2000) 35-49.
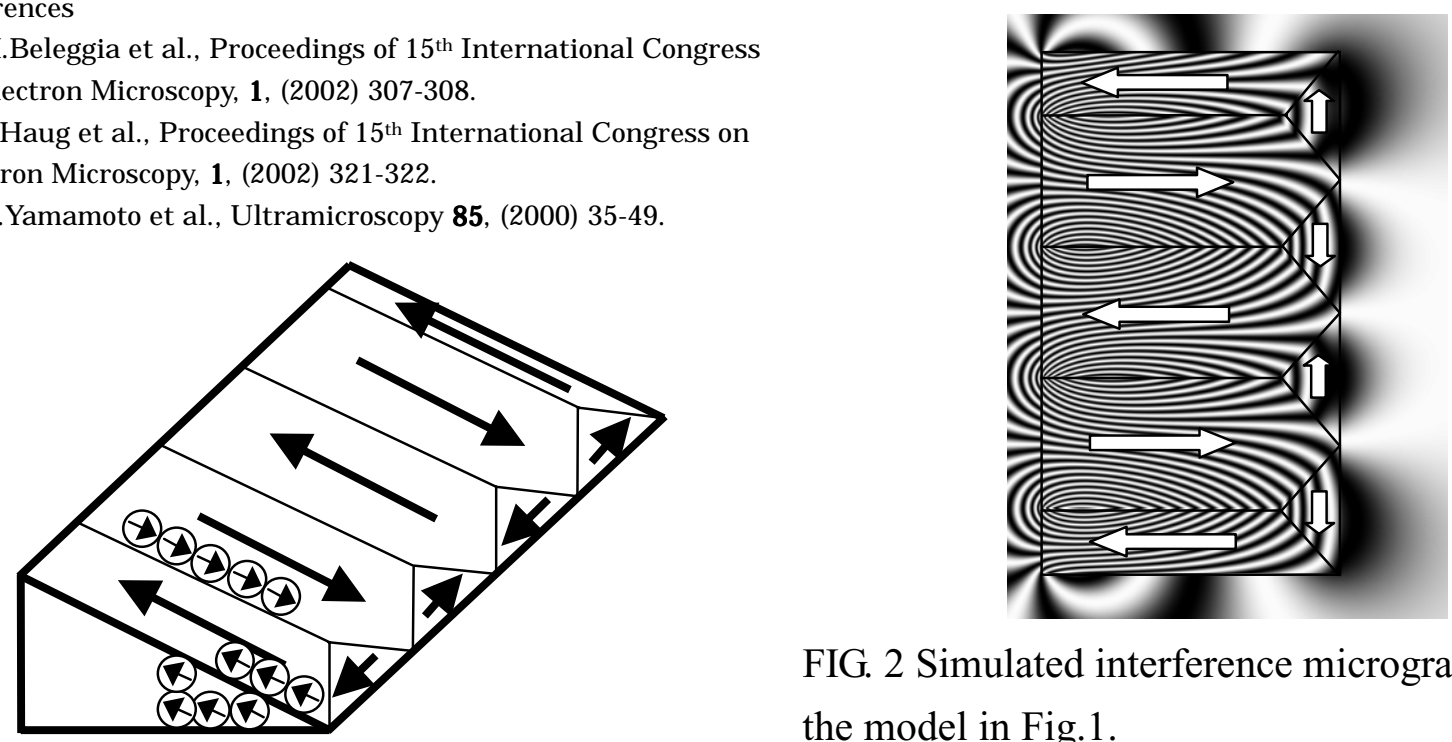

FIG. 2 Simulated interference micrograph of the model in Fig.1.

FIG. 1 Model of a wedge-shaped magnetic specimen.
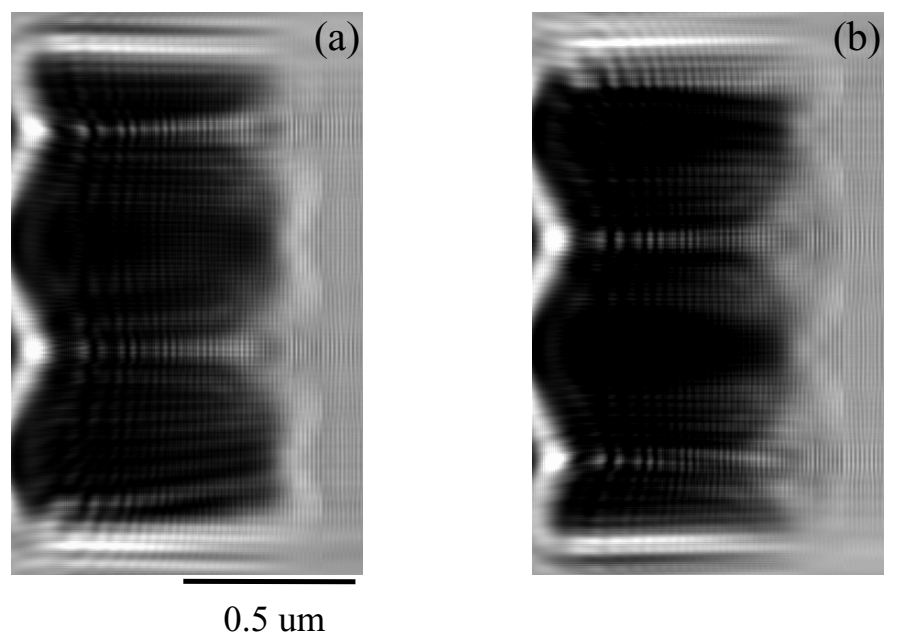

FIG. 3 Simulated Lorentz images of the model in Fig. 1, (a) over focused, (b) under focused.

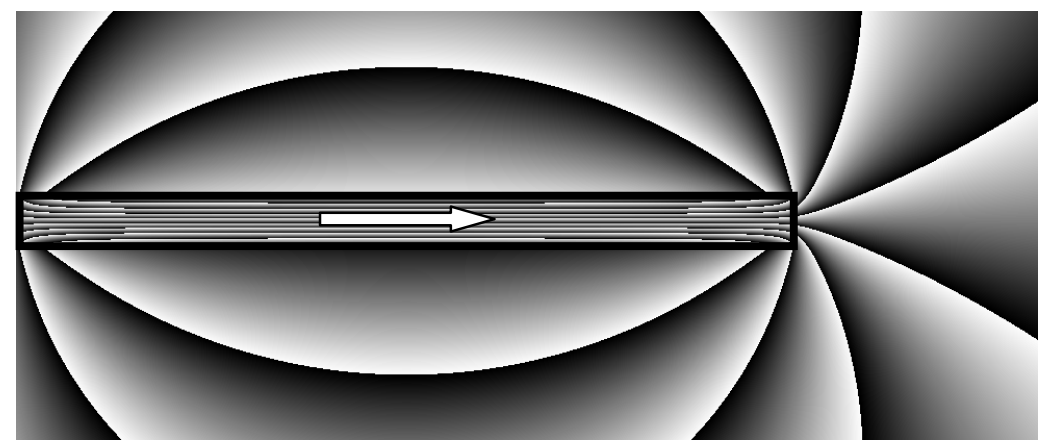

FIG. 4 Simulated phase image of a cylindrical magnetic specimen. 